\title{
Treatment for ADHD and Their Effectiveness
}

\author{
Diyun Zheng \\ Nanjing Foreign Language School International Centre, Nanjing, Jiangsu 210046, China \\ *Corresponding author. Email: 2351215032@qq.com
}

\begin{abstract}
Attention Deficit Hyperactivity Disorder is a chronic neurodevelopmental disorder that often appear on preschoolers or school-age children. Main treatment of the disorder involves drug therapy and non-drug therapies, the different treatments have different effects and adverse reactions. This paper looks into the mechanism of action of some most commonly used treatments like CNS and non-CNS stimulants, psychosocial therapies and NIBS, as well as the extent to which they are effective. This paper also attempted to discuss which is the most effective among them. The discussion comes to a result that all therapies listed are not perfect and can still be improved. To know about the imperfections of these cures will help future studies to develop more complete, more effective and less harmful therapies to help patients overcome their ADHD disorder.
\end{abstract}

Keywords: Attention Deficit Hyperactivity Disorder (ADHD), Psychosocial Therapy, Non-Invasive Brain Stimulation (NIBS)

\section{INTRODUCTION}

Attention Deficit Hyperactivity Disorder is a chronic neurodevelopmental disorder that is commonly seen in children and is characterized by inattention, hyperactivity and impulsive behavior, which can lead to cognitive and behavioral deficits, anxiety, depression, marital and occupational problems, and have become an important public health problem.

ADHD usually starts in pre-school age and school age, and the symptoms can last until adulthood, which can have negative effects on children's study, family life, social adaptation and other aspects. Therefore, early recognition and intervention of ADHD children and alleviation of behavioral problems are of great importance to children and families.

Some often used therapies include using medicine like methylphenidate (MPH) and lisdexam fetamine dimesylate (LDX) or non-drug therapy involving psychosocial therapy, non-invasive brain stimulation(NIBS), neurofeedback(NF), cognitive treatment $(\mathrm{CT})$.

This literature review will look into some of the various treatment of ADHD, mainly non-drug therapies, and try to find out how effective the different therapies are.
The information is searched through the internet to find relevant research articles about ADHD and its cure.

The data was extracted using a standardized form.

Information found showed that researchers have not yet come to a clear conclusion on which therapy exactly works the best, but the common knowledge is that different methods are suitable for different age group and intelligence level.

\section{DRUG THERAPY}

Drug treatment of ADHD includes central nerve system (CNS) stimulants and non-CNS stimulants.

Mechanism of action of CNS stimulants, for example Methylphenidate (MPH), is that the stimulants block the neuron's reuptake of dopamine and norepinephrine, promoting the release of these monoamines into the neuronal interstitial spaces, therefore achieve therapeutic effects. Non-CNS stimulants, such as lisdexam fetamine dimesylate(LDX), regulate responses to external stimuli by blocking the reuptake of norepinephrine and dopamine, increasing the concentration of norepinephrine and dopamine between synapses and inhibiting catabolic metabolism, thus manipulate the respond to external stimuli.[1,2] 


\section{PSYCHOSOCIAL THERAPY}

Non-drug therapy includes psychosocial therapies, neurofeedback, cognitive training, non-invasive brain stimulation, etc. Among these, psychosocial therapies include cognitive behavioral treatment (CBT), behavioral parental treatment (BPT), and behavior intervention training, etc.

\subsection{Cognitive Behavioural Treatment}

For CBT, it is a combination of cognitive treatment and behavioral treatment. Cognitive therapy uses cognitive restructuring to improve children's behavior, while behavioral therapy focuses on using principles of classical conditioning and imitation learning to correct bad behavior as well as to reinforce positive behavior. Most CBT training pay more attention to behavioral instead of cognitive treatment. Researchers applied adult CBT treatment containing cognitive therapy to adolescents and find it successful as the symptoms are improved and the anxiety as well as depression that usually appear along with ADHD are released.[2]

\subsection{Behavioural parental treatment}

Behavioral parental treatment (BPT) is the therapy that instructs parents. The therapy guides parents to correctly understand the disease, it teaches parents to use means like demonstration, discussion and role playing in order to improve the management ability of ADHD children and therefore bad behaviour of children can be reduced, the parent-children relationship can also be improved. Parents can promote the development of positive behavior in children, yet may also unintentionally cause a negative impact, which may exacerbate the child's behavior problems. Therefore, it is very important to establish healthy parent-child interaction for the growth of children, which is what BPT pays a great amount of attention on. BPT can also work by improving parental adherence to medication and thus increase efficacy. Among different BPT programs, four are the most commonly used:

\subsection{1. $3 P$}

Positive Parenting Program (3P), the Incredible Years (IY), Parent-Children Interaction Therapy (PCIT), New Forest Parenting Program (NFPP).

Positive parenting Program is proved to be effective by researchers. The research included 48 subjects and mothers are randomly divided into two equal groups. The mothers of the first group participated in the 3P program, while the second group did not receive any treatment. ADHD symptoms, mood, family function and parental attitude were compared between the two groups before and after the program. The results showed that $3 \mathrm{P}$ could be useful treating ADHD children.[3]

\subsubsection{IY}

In terms of the Incredible Years program, a research made by Margiad E et.al. (2020). chose 58 families, 29 attended the program while the rest 29 families belong to the controlled group. The feasibility and acceptability of the program received positive feedback from parents of ADHD children.[4]

\subsubsection{PCIT}

Parent-Children Interaction Therapy include two phases, which are children-directed interaction (CDI) and parent-directed interaction (PDI) respectively. In CDI stage, therapists teach parents how to better interact with their ADHD children. The aim is to improve children's self-esteem, enhance parent-child attachment and strengthen children's prosocial behaviors.[2]

Interactive games have a purpose of improving children's self-esteem and enhance parent-child attachment

Strengthening children's prosocial behavior. In this phase parents learn to use a technique named PRIDE, consisting of Praise, Reflection, Imitation, Description, and Enthusiasm. Praise stands for complimenting children's good behaviour verbally; reflection means to give response to children's oral expressions; imitation represents games where parents imitate the child; description is to describe children's proper behaviour and enthusiasm means that parents need to be encouraging when, for example, praising children's accomplishment. During PDI period, parents are instructed by the therapist to give clear, proper, direct instructions and give appropriate feedback according to children's respond to the instructions that parents give.[5]

There are some special techniques used in PCIT, for instance On-site real-time guidance is used during the therapy, which means that the therapist watches parental-children interactions through one-way glass and give parents instructions real-time. Its unique advantage lies in the immediacy of feedback. Parents can learn proper parenting and interaction skills through the real-time feedback of the therapist, and any questions can be answered and guided by the therapist during the training period. In addition, PCIT focuses on the interaction between parents and children. Parental affection has a great influence on children's mood and behavior, especially young children.

PCIT programs influences children with several different techniques. Behavioral description helps ADHD children maintain attention. Parent-child attachment theory is also applied in the training process to improve parent-child relationship and teach parents to play a positive guiding role in improving children's behaviour. PCIT also pay attention to children's 
emotional regulation (ER) abilities. In recent years, some studies have reached a conclusion that children with ADHD are more deficient in ER. Abnormal emotional regulation may be an important reason for ADHD children's inattention, hyperactivity, impulsiveness and other behavioral manifestations as well as functional impairment. Many scholars believe that abnormal emotional regulation itself is a core symptom of ADHD patients (Zhang Lin et al. (2021)).

\subsection{Behaviour Intervention Training}

Behavior Intervention Training is one type of psychosocial therapies and it mainly include 5 steps. First step is Self-control Training. Self-control training is a method of behavior modification carried out by children themselves. It is characterized by the children themselves taking the main responsibility in the process of changing behavior. There are two main types of selfcontrol training: 1. Learning to curb excessive behaviors that bring immediate satisfaction, such as uncontrolled lifestyle, overeating, smoking, drinking, and addiction to television and game consoles. 2. Making efforts to increase certain good behaviors, for instance, develop study habits, physical exercise, building self-confidence, etc. The process of self-control training includes three basic steps: self-discipline, self-recording and selfimplementation of immediate consequences. The second step is to do relaxation training through muscle relaxation or use biological feedback as directed. The third step is Behaviour Modification. In this step methods of operant conditioning are applied. Positive and negative reinforcement or punishment are used to shape ADHD children's behaviour. The fourth step is Catharsis Therapy which origins from psychoanalysis treatments. It lets children to express their negative emotions towards parents or things, additionally, it Instruct children to do outdoor and physical training activities to release energy. The fifth step is Family Therapy. Family therapy requires parents to find the good about the child, and through rewarding and mild punishment to establish children's self-esteem and selfconfidence; it also requires parents and children to learn together, and to explore solution to the problems through communication, thus create a good family atmosphere[6]

\subsection{NIBS}

Non-Invasive Brain Stimulation (NIBS) is a technology that is used in treating ADHD. Two main types of NIBS can be applied to ADHD cure, namely, Transcranial Direct Current Stimulation(tDCS) and Transcranial Magnetic Stimulation (TMS). In this article the more commonly used one, which is tDCS, would be introduced.

Transcranial Direct Current Stimulation applies a weak direct current to certain areas of the brain via electrodes attached to the scalp. tDCS does not directly induce brain activity, but changes spontaneous brain activity excitability through subliminal modulation of neuronal membrane potential. The effects of stimulation are determined by the polarity and areas of the brain that the current applies to. In terms of polarity, the anode increases neuronal excitability and the cathode decreases it. tDCS can produce long-term potentiation (LTP) or long-term depression (LTD) of synaptic excitability by regulating synaptic plasticity, and the efficacy can reach more than 24 hours, which actively demonstrates that it has persistent regulation of neuroplasticity. Compared to other NIBS technologies such as TMS, tDCS has advantages over portability, economic suitability, tolerability and safety. For these particular reasons stated above, tDCS is what a greater number of families chose as treatment to their children's ADHD.[7]

\subsection{Other}

Some other cure to the disorder of ADHD include Neurofeedback (NF), Cognitive Training (CT), nondrug therapy of TCM (Traditional Chinese Medicine), etc. [2]

Neurofeedback is a technology that manipulatively modulates brain activities and turns biological indicators into intuitive visual or auditory information to help patients change their states of mind as well as their behaviour through training and, as a result, improve biological indicators and clinical symptoms. Neuron feedback include three main types, electroencephalogram neurofeedback (EEG-NF), nearinfrared spectroscopy neurofeedback (NIRS-NF), fMRIbased neurofeedback (fMRI-NF).

Cognitive treatment is therapy that improves ADHD children's cognition and behaviour based on the theory of brain plasticity.

\section{DISCUSSION}

\subsection{Drug}

Adverse reactions to CNS stimulants include sleep disturbance, appetite suppression and epileptic twitching, while for non-CNS stimulants the adverse reactions include loss of appetite, headache, insomnia and irritability.

CNS stimulant is currently the most preferred type of drug for clinical treatment of ADHD. The particular reason for the circumstance is the high clinical response rate at a percentage of $80 \%$. However, there are still a significant number of patients that do not respond to CNS treatment, which actively demonstrates the importance of development and application of non-CNS stimulants. For instance, Atomoxetine, the only nonCNS drug approved by FDA in recent years, has showed a significant effect on various core symptoms of ADHD in children and adults. 


\subsection{BPT}

For preschoolers, BPT can effectively improve the core symptoms of ADHD, and the effect is better than drug intervention. BPT is recommended by AAP, CPS and NICE guidelines as the preferred treatment for preschool children with ADHD, while in terms of school-age children and adolescents, parental assessment suggest that BPT can improve the main symptoms of ADHD, whereas evaluation based on blind method showed that the improvement of core symptoms was not obvious, which might be related to the enhancement of self-consciousness and prone to rebellion in teenagers and school-age children.[2]

\subsection{BIT}

The use of Behavioral Intervention Therapy can effectively stimulate the development of the brain, and can help the reconstruction of the brain conditioning system, and then play a role in enhancing the cognitive ability of children, stabilizing children's mood, improving children's symptoms and enhancing children's attention. Factors that may affect the effectiveness of the therapy include the severity of symptoms, level of intelligence and age. The more serious the disorder is the harder to treat by Behavioral Intervention Therapy and children with lower intelligence level or at a younger age are also more likely to find the therapy not helpful.[6]

\subsection{NIBS}

Non-Invasive Brain Stimulation can improve the behavioral inhibition and executive function of ADHD patients, improve the accuracy of working memory and response speed. However, due to the complex interaction mechanism between brain regions, target and parameter setting will affect the therapeutic effect of target symptoms, which is a reason why NIBS need to be applied clinically with great caution.[7]

During NIBS therapies, patients often face the problems of limited drug selection and high rates of adverse reactions including headache, neck pain, mild drowsiness, skin tingling or itching, burning sensation and redness of skin, whereas tDCS provides a new treatment option for these patients, so it is of great significance to carry out relevant clinical studies.[8]

\section{CONCLUSION}

Attention Deficit Hyperactivity Disorder Is a disorder that may have serious effects to patients' future life if not cured early, therefore effectiveness of different therapies should be seriously considered before applying to patients. For instance, the intelligence level and severity of disorder should be evaluated before using behavioral intervention therapy and adverse reaction of drugs as well as NIBS should also be taken into consideration to make sure children are not harmed during the process of treatment. More effective and less harmful ways should be discovered and used practically. Effective treatment not only benefits the patient himself, but also the entire society. Future studies should focus on reducing side effects of treatment and try to develop new methods that are less harmful to patients, as well as to apply them in clinicals.

\section{REFERENCES}

[1] He, L. \& Zhang, Y.P.(2020). Progress in evidencebased research on attention deficit hyperactivity disorder in children. doi:CNKI:SUN:SDYY.0.2020-07-005.

[2] Zhu, Y. K., Wang, X., Wang, L. \& Yang, J.(2020). Research progress in non-pharmacological treatment of attention deficit hyperactivity disorder in children. Doi :CNKI:SUN: YYCY.0.2020-30009.

[3]Öztürk Yusuf and Özyurt Gonka and Akay Pekcanlar Aynur. (2019). The effects of the triple Ppositive parenting programme on parenting, family functioning and symptoms of attention-

deficit/hyperactivity disorder. A randomized contro lled trial. Psychiatry and Clinical Psychopharmacol ogy, 29(4), pp. 665-673.

[4] Margiad E. Williams and Richard P. Hastings and Judy Hutchings. (2020). The Incredible Years Autism Spectrum and Language Delays Parent Program: A Pragmatic, Feasibility Randomized Controlled Trial. Autism Research, 13(6), pp. 1011-1022.

[5] Zhang, J., Tian, L., Shi, H. X., Zhang, Y. N. \& Zhang, L.(2021). Effect of parent-child interaction therapy on preschool children with ADHD. Doi :10.19347/j.cnki.2096-1413.202102066.

[6] Wei R Z. (2021). Influencing factors of behavioral intervention training in the treatment of children with hyperactivity disorder. Chinese and foreign medical research (20), 194-196. The doi: 10.14033 / j.carol carroll nki CFMR. 2021.20.068.

[7] Qian, N. S., Hong, W. \& Li, C. B.(2021). Research progress of transcranial direct current stimulation in the treatment of children and adolescents with mental disorders. Journal of Shanghai Jiao Tong University (Medical Edition)(), doi:10.3969/j.issn.1674-8115.2021.09.001.

[8] Fan, Z. \& Li, Y. (2008). Non-central stimulant therapy for attention deficit hyperactivity disorder. Doi: $\quad$ CNKI:SUN: LCAk.0.2008-10-033. 